\title{
A review on the cell biology and toxicology within the correlation to a new achievement
}

\author{
DuraidHamid Naji ${ }^{(1)}$, MohammedBadran ${ }^{(2)}$ \\ School of Life Science Huazhong University of Science and Technology, Wuhan, China, 430074
}

The mixing of cell biology within the toxicology reveals the potential impacts on the elements involving cellular structures, organelles, functioning characteristics, metabolic circles, signaling pathways, as well as interactions within the microenvironment. These mentioned elements are not only involving chemicals and drugs, but one should also put into consideration other factors like food, water, as well as the air we inhale. Therefore, a level of toxicology subspecialties have been developed and will be lately defined within the development of biotechnologies and the latent understanding of toxicogenomics and ecotoxicology or aquatic, chemical, clinical, environmental, forensic, medical, occupational, and regulatory toxicology.

According to that, future issues of the journal will concentrate on both the clinical and translational work through an emphasis on the molecular in a cell biology, genetic and epigenetic heterogeneity, drug discovery and development, and molecular addition to the merger of cell biology and toxicology that enables a deeper insight of human regulation, cell type-specific function, and system biomedicine in drug discovery and development $(1,2)$. It is well known and accepted that "adverse effects" or "toxic impacts" of elements from extracellular, intercellular, or intracellular resources contribute to the pathogeneses and exacerbations of diseases. Within the application of this process, we explore gene- and protein-based regulations by the usage of genomics and proteomics, functional genomics and proteomics, computational biology, and gene expression and function analysis.

There is no doubt that the combination of cell biology and toxicology is a major and important approach to reveal multifactorial genetics and epigenetics of diseases to develop the strategy of precision medicine and disease-specific biomarkers to figure out the deterioration of the disease and efficiency of therapies, developmental biology, and system biology to understand multi- principles of element actions or bioinformatics-based data mining approaches to paint the full picture of the disease. Even though, there is a remarkable lack in our findings concerning the existence of the disease per se can reduce or increase the cell sensitivity and response to elements. As an example, astrocytes as central nervous system glial cells in the disease change their biological behaviors and functions, leading to the disorder of extracellular ion and neurotransmitter exchanges and dysfunction of the blood-brain barrier (3). Pathological astrocytes will alter the diffusion speed of elements and reduce resistance of nervous cells, promoting and maintaining the "side effect."

The impact of mixing the study of cell biology and toxicology to help in the identification of molecular targets during discovery and development of target-based drugs should be highly considered. Drug target identification and validation is considered to be one of the most important procedures during drug development and decides the processes of rational drug design, genetic/small molecule (high-throughput) screening, novel therapeutic approaches, combinatorial and parallel synthesis of drugs, pharmacokinetics and pharmacodynamics, pharmacogenomics, or toxico-genomics. Precision medicine has been recommended as a new study to discover and develop medicines and vaccines within the integration of the clinical and molecular information on molecular mechanism of diseases (4) to a wisely select disease-specific targets and identify patient populations and to enhance clinical outcomes.

The identification of disease-specific goals is of paramount consideration in the development of precision medicine in order to obtain clinically significant treatments with sufficient safety profiles. Even though, the behavior of targeting molecules does not reveal a simple as high or low expression levels, with or without mutations, or simply active or not. As an example, CD5L/AIM was recognized as a regulator expressed in the non-pathogenic, without including pathogenic Th17 cells by single-cell RNA-seq (5). CD5L has the ability toact as a functional switch to regulate Th17 cells from the normality to the pathogenicity leading to the occurrence of autoimmunity, however CD5L could not change Th17 differentiation. Amongst, Dolsten and Søgaard(6) suggested that the drug effect should be the key point of driving factor in order to ensure the progress of precision medicine. In addition, a number of important risk impact factors in the development of disease and mortality call for special consideration from scientists when we evaluate the toxic influences of elements. Additionally, elements per se, the combination of formulations, delivery forms and schedules, sleep-wake cycles, physical activities, related organ functions, sensitivity/resistance, lifestyle, or gut microbiome may add more on the variants between individuals, organs, cells, or molecules. 
The effect of cell biology and toxicology on biological function- and disease-specific biomarkers not only for identification of drug efficacy and efficiency but also for detecting drug toxicity and toxicology should be further considered. Disease-specific biomarkers have been recommended as one of the five critical factors in the practice of precision medicine to monitor the quality of therapeutic strategies, to detect patient response to targeted treatments, and to predict the prognosis of disease $(7,8)$. Dynamic changes of complex interaction networks and molecular subnetworks can represent and influence responses of cells or organs to real-time changed elements. We should pay even more attention on the identification and validation of biomarkers, network biomarkers, and dynamic network biomarkers to monitor drug resistance, toxicity or toxicology, by integrating bioinformatic-based screening with clinical informatics and phenotypic information $(9,10)$. The rewiring of module networks is used to characterize functional reorganization of a complex biological function and to study the dynamical sensitivity and resistance of drugs. Consistent module genes can be directly used to reveal new genotypes relevant to drug sensitivity, different from gene expressions. We suggest the hierarchical structures of the temporal module network as spatio-temporal biomarkers to monitor the efficacy, efficiency, toxicity, and resistance of the therapy.

We have put into consideration the impact of genetic and functional alterations of membrane biology and domains or associated receptor- and non-receptor-mediated signaling and transport proteins of the cell membrane, mitochondria, endoplasmic reticulum, Golgi apparatus, and other organelles in health and disease. Also, it is important to investigate the influence of gene mutations, epigenetics, RNA profile changes (e.g., microRNA, circular RNA, long-noncoding RNA), DNA sequencing, or heterogeneity (11) in order to understand cancer development, maintenance and treatment resistance, immune and inflammatory cell function, neuroscience, stem cell development and differentiation, epithelial biology, and endothelial barrier function in toxicology of elements inducing carcinogenesis or treating cancer. For example, microRNAs control gene expression post-transcriptionally, are released upon cellular damage, and act as epigenetic regulators or potential non-invasive biomarkers of injury (12). It is envisaged that combining cell biology and toxicology will make great contributions in the discovery and validation of miRNA biomarkers, provide an insight into injury mode of action, as well as, help to identify specific injury locations and affected cell types. Biological function- and disease-specific biomarkers should have a global standard for practice of toxicity and toxicology, for clinical validation, or for safety assessment. With the development of deep-sequencing approaches, altered lengths of miRNAs were suggested as critical factors regulating the processing to fine-tune miRNA target specificity (13). The altered miRNA seed sequence could guide strand selection to increase the number of targets a single pre-miRNA may eventually repress.

We should consider the effects of toxic elements produced from host cells during stress, injury, and trauma. Eosinophils play critical roles in the maintenance of innate immunity and the pathogenesis of various inflammatory and allergic disorders. During inflammatory responses, eosinophils can generate secretory granules which contain the eosinophil major basic protein, one of four cytotoxic proteins. Such protein toxicity can be activated by granule acidification and aggregated in extracellular microenvironment to mediate the damage to pathogens and regulate toxicity intra- and extracellularly. This is important for innate immunity and immunopathology through its polymorphic self-association pathways. The overproduction of those toxic proteins may increase the sensitivity of cells; amplify toxic effects of elements in the immune system, central and autonomic nervous systems, gastrointestinal system, cardiovascular and circulatory system, endocrine system, pulmonary system, skeletal and muscular system, and urinary system; and help us to further understand their pathogeneses and therapies. In addition, we should consider the heterogeneity between individuals, organs/tissues, locations within the tumor, or cells within the location to respond to toxic elements. Therefore, it is the time to highly consider the correlation between cell biology and toxicology. We have remarkable ways to figureout the alterations of spatial genome organization and their influences on transcription in cell toxicology, adopt the developed chromatin interaction analysis within the paired-end tag sequencing strategy, or understand higher-order chromosome folding and specific chromatin interactions in the topological mechanism of human variation and disease induced by several toxic elements. We declare that single-cell genomics and biology will aid in understanding the cell heterogeneity and response to elements, the molecular underpinnings at a genomic scale, or the molecular mechanisms for the heterogeneity and pathogenicity of toxic elements.

\section{REFERENCES}

[1] Abraham E, Marincola FM, et al. Clinical and translational medicine: integrative and practical science. ClinTransl Med. 2012;1:1.CrossRefPubMedPubMedCentralGoogle Scholar

[2] Alam MM, Lal S, FitzGerald KE, Zhang L. A holistic view of cancer bioenergetics: mitochondrial function and respiration play fundamental roles in the development and progression of diverse tumors. ClinTransl Med. 2016;5:3.CrossRefPubMedPubMedCentralGoogle Scholar

[3] Chen $\mathrm{C}, \mathrm{He} \mathrm{M}$, et al. Five critical elements to ensure the precision medicine. Cancer Metastasis Rev. 2015;34(2):313-8.CrossRefPubMedGoogle Scholar 
[4] Dolsten M, Søgaard M. Precision medicine: an approach to R\&D for delivering superior medicines to patients. ClinTransl Med. 2012;1:7.CrossRefPubMedPubMedCentralGoogle Scholar

[5] He MY, Xia JL, et al. The development of precision medicine in clinical practice. ClinTransl Med. 2015;4:28.CrossRefPubMedCentralGoogle Scholar

[6] Montgomery TA, Ruvkun G. MicroRNAs visit the ER. Cell. 2013;153(3):5112.CrossRefPubMedGoogle Scholar

[7] Soragni A, Yousefi S, et al. Toxicity of eosinophil MBP is repressed by intracellular crystallization and promoted by extracellular aggregation. Mol Cell. 2015;57(6):1011-21.CrossRefPubMedGoogle Scholar

[8] Tangye SG, Brink R, et al. SnapShot: interactions between B cells and T cells. Cell. 2015;162(4):9266.CrossRefPubMedGoogle Scholar

[9] Wang C, Yosef N, et al. Single-cell genomics unveils critical regulators of Th17 cell pathogenicity. Cell. 2015;163(6):1400-12.CrossRefGoogle Scholar

[10] Wang XD, Marincola FM. A decade plus of translation: what do we understand? ClinTransl Med. 2012;1:3.CrossRefPubMedPubMedCentralGoogle Scholar

[11] Wu XD, Chen LN, et al. Network biomarkers, interaction networks and dynamical network biomarkers in respiratory diseases. ClinTransl Med. 2014;3:16.CrossRefPubMedPubMedCentralGoogle Scholar

[12] Yates LA, Norbury CJ, et al. The long and short of microRNA. Cell. 2013;153(3):5169.CrossRefPubMedGoogle Scholar

[13] Zeng T, Wang DC, et al. Prediction of dynamical drug sensitivity and resistance by module network rewiring-analysis based on transcriptional profiling. Drug Resist Updat. 2014;17(3):64-76. 